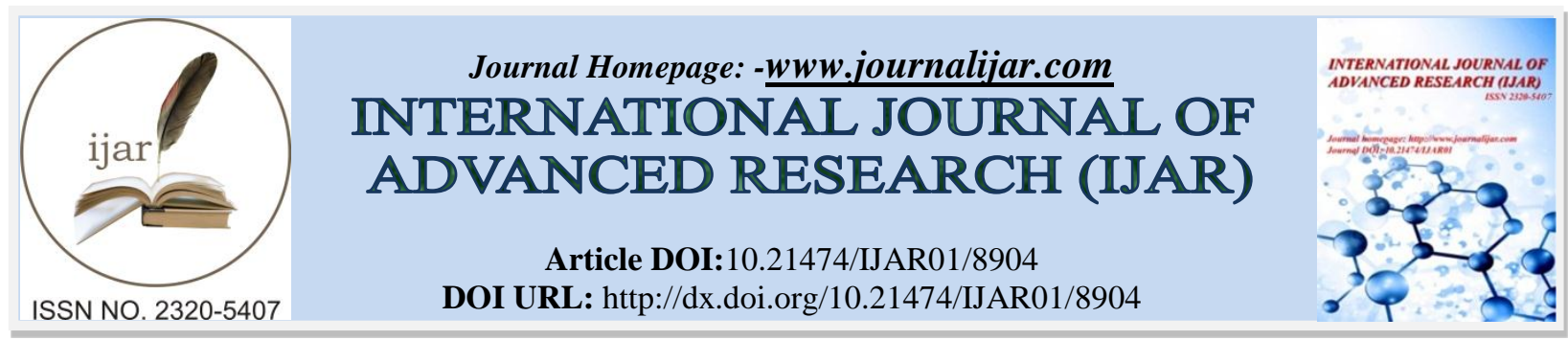

RESEARCH ARTICLE

\title{
STUDY OF BACTERIAL PATHOGENS WITH THEIR ANTIBIOGRAM CAUSING DIABETIC FOOT ULCERS.
}

\author{
Shanthi sree Vaddadhi ${ }^{1}$, Dr. P. Ratna Kumari ${ }^{2}$ and Dr.R.Lakshmi Kumari ${ }^{3}$. \\ 1. $2^{\text {nd }}$ year Post Graduate, Department of Microbiology, Siddhartha Medical College,Vijayawada-520008. \\ 2. Professor ,Department of Microbiology, Siddhartha Medial College,Vijayawada-520008. \\ 3. Head of the Department, of Microbiology, Siddhartha Medical College,Vijayawada-520008.
}

\section{Manuscript Info}

\section{Manuscript History}

Received: 12 February 2019

Final Accepted: 14 March 2019

Published: April 2019

Key words:-

Diabetes mellitus, ulcers, infections, complications.

\begin{abstract}
Globally and nationally, Diabetes mellitus with its complications has become the contemporary and challenging health problem. Diabetic foot ulcers are the most serious complication of the disease. Diabetes mellitus appears to be an independent risk factor for infection with multi drug resistant organisms causing several abnormalities of the host defense mechanisms that might result in a higher risk of certain infections. Hence early diagnosis of microbial infections will aim to appropriate antibiotic therapy to avoid further complications such as cellulitis, gas gangrene and amputation. Hence this study had been done to evaluate the microbial pathogens causing diabetic foot ulcers along with its antibiogram. Pus samples were collected from 100 patients with diabetic foot ulcers attending Department of Surgery, Government General Hospital, Vijayawada and sent to Microbiology department, Siddhartha Medical College for processing. Diabetic patients of all age groups and both gender were included and those patients who were on antibiotic therapy were excluded. Out of 100 samples processed 90(90\%) were culture positives, of which 101 pathogens were isolated, 70(69.3\%) were Gram negative and $31(30.6 \%)$ were Gram positive Pseudomonas ssp were found to be predominant followed by Escherichia.coli and Staphylococcus aureus. All the pathogens were found to be highly sensitive to Piperacillintazobactam, Gentamicin and least sensitivity to Azithromycin. The results of this study therefore alert us to the need for proper management of antibiotics to optimize patient care and improve clinical outcome.
\end{abstract}

Copy Right, IJAR, 2019,. All rights reserved.

\section{Introduction:-}

Diabetes is a metabolic disorder which affects, around one million people nationwide, it is often accompanied by serious complication and still today there is no cure yet. Globally and nationally, Diabetes mellitus with its complications has become the contemporary and challenging health problem.

Corresponding Author:-Dr. P. Ratna Kumari

Address:- Professor, Department of Microbiology, Siddhartha Medical College,Vijayawada520008 . 
Diabetes mellitus appears to be an independent risk factor for infection with multi drug resistant organisms causing several abnormalities of the host defense mechanisms that might result in a higher risk of certain infections. Diabetic foot infections include cellulitis, abscess, necrotizing fasciitis, septic arthritis, tendonitis and osteomyelitis. Diabetic foot ulcers are the most serious complication of the disease. Hence early diagnosis of microbial infections will aim to appropriate antibiotic therapy to avoid further complications such as cellulitis, gangrene and amputation. If not treated promptly sometimes infection leads to amputation of foot.

\section{Methods:-}

The study was conducted for a period of 8 months (May-Dec 2018) in Government General Hospital, Vijayawada on 100 Diabetic patients with infected foot ulcers who were admitted in surgical unit of Government General Hospital, Vijayawada. To avoid contamination, specimens were collected under aspectic condition and samples were transported to Microbiology department in Siddhartha Medical College. Samples were subjected to direct Gram staining and then inoculated on Nutrient agar, Blood agar, MacConkey agar. Isolates were identified and confirmed by biochemical reactions. Antibiotic susceptibility testing was performed by Kirby Bauer Disk Diffusion method as per CLSI (2017) guidelines. Gram positive isolates were tested for Ampicillin, Piperacillin-tazobactam, Cefoxitin, Cephalosporins, Gentamycin, Ciprofloxacin, Vancomycin, Linezolid. Gram negative isolates were tested for Piperacillin-tazobactam, Cepahalosporins, Azithromycin, Levofloxacin, Cotrimoxazole, Imepenem.

\section{Results:-}

Out of 100 samples processed 72 were from males \& 28 samples were from females, and male to female ratio was 2.6:1. Majority of the patients in the present study were more than 51 years i.e. 33(33\%) being in the 51-60 years of age, 29(29\%) in between the age of 41-50 years and 25(25\%) in > 61 years of age. Diabetic foot complications manifests in myriad forms and in this study it was observed that 50(50\%) were with ulcer foot, 22(22)\% patients were presented with cellulitis, 14(14\%) with abscess, 10(10\%) with gangrene of one or more toes, and $4(4 \%)$ with necrotizing fasciitis

.Out of 100 samples processed 90(90\%) were culture positive.Out of 90 culture positives, $89 \%$ ( $\mathrm{n}=80$ ) yielded monomicrobial growth and $11 \%(\mathrm{n}=10)$ with polymicrobial growth. Out of 101 pathogens isolated, 70(69.30\%) were Gram negative and 31(30.6\%) were Gram positive. Pseudomonas aeruginosa 23(23\%), was most common isolate causing diabetic foot infections, followed by Escherichia coli 19(19\%), Staphylococcus aureus 18(18\%), Klebsiella spp.,15(15\%), Proteus sp., 11(11\%), CONS 10(10\%), Enterococci 3(3\%) and Citrobacter $2(2 \%)$.

Out of 23(23\%) Pseudomonas isolates, 22(95\%) were sensitivity to Piperacillin tazobactam. 13(56\%) were sensitivity to Gentamicin and Only 2(8\%) least sensitive to Azithromycin. Out of 19(19\%) E.coli isolates $15(78 \%)$ were sensitive to Gentamicin and $12(63 \%)$ were sensitive to Piperacillin tazobactam and 2(10\%) least sensitive to Azithromycin. Out of $15(15 \%)$ Klebsiella isolates $12(80 \%)$ were sensitive to Piperacillin tazobactam, least sensitive $1(1.66 \%)$ to Azithromycin. Out of $11(11 \%)$ Proteus isolated, $9(81 \%$ )were sensitive to Piperacillin tazobactam, 3(27)\% to Gentamicin and least sensitivity 2(18\%) to Levofloxacin. All the Citrobacter isolates were $100 \%$ sensitive to Piperacillin tazobactam.

Out of 31(30.6\%) Gram positive organisms isolates, 18(18\%) were Staphylococcus aureus of which 12( 66.6\%) were sensitive to Vancomycin, Linezolid, Piperacillin tazobactam and low sensitive 1(33.3\%) to Cefapaerazone Salbactam. Out of $10(10 \%)$ CONS $8(80 \%)$ were sensitive to Piperacillin tazobactam and least sensitive 1(10\%) to Cefaperazone salbactam. Entericocci isolates were sensitive to 3(100\%) Piperacillin tazobactam.

\section{Discussion:-}

Most of the patients in this study belonged to age group 51-60years, which is in accordance to studies by Patil SV et al, Jain \& Barman et al, and Khare $\mathrm{J}$ et al.Age distribution: In this study, males were more prone for Diabetic foot lesions compared to females with M:F ratio 2.5:1 this is in relevance to the study done by Khare $\mathrm{J}$ et al who has shown M:F ratio 2.6:1. Higher male prevalence is seen in some other studies like Jain \& Barman et al and Shareef et al with M:F ratio 9:2 \& 6.1:2 respectively. $90 \%$ of the samples has shown culture positivity, Out of which $89 \%$ yielded monomicrobial and $11 \%$ yielded polymicrobial growth which is comparable to the study done by Khare $\mathrm{J}$ et al i.e., $90.4 \%$ and $9.6 \%$ respectively. Out of 101 pathogens isolated $69.3 \%$ were Gram negative and $30.6 \%$ were Gram positive organisms, this is in relevance to the study of Shareef et al with $64.75 \%$ Gram negative and study by Patil SV et al with $30.55 \%$ Gram positive organisms. 
The study showed a preponderance of gram-negative bacilli among the isolates from the diabetic foot ulcers, hence all patients with Diabetic foot infections admitted to a tertiary care hospital in India may not require empirical therapy for Gram-positive coverage. A $\beta$-lactum agent with/without inhibitor combination or a quinolone as an empirical agent after establishing the patients history of previous antibiotic usage would probably be more appropriate. In the event of the Pseudomonas infection, an anti-pseudomonal drug can be added. In the present study, Piperacillin-tazobactam/Cefaperazone salbactam adequately covered such infection. The lack of multidisciplinary approach in the treatment of diabetic foot is quite obvious and there is a lot of scope of improvement in the form of holistic approach to a patient with diabetic foot rather than just treating the ulcer foot. The results of this study therefore alerts us to need for proper management of antibiotics to optimize patient care and improve clinical outcome.

\section{Acknowledgement:-}

I am thank full to all the doctors, technical staff of Microbiology Department, Government General Hospital, Siddhartha Medical College,Vijayawada who cooperated us in carrying out this research work

Fig no 1:-Sample Collection \& Diabetic foot ulcer
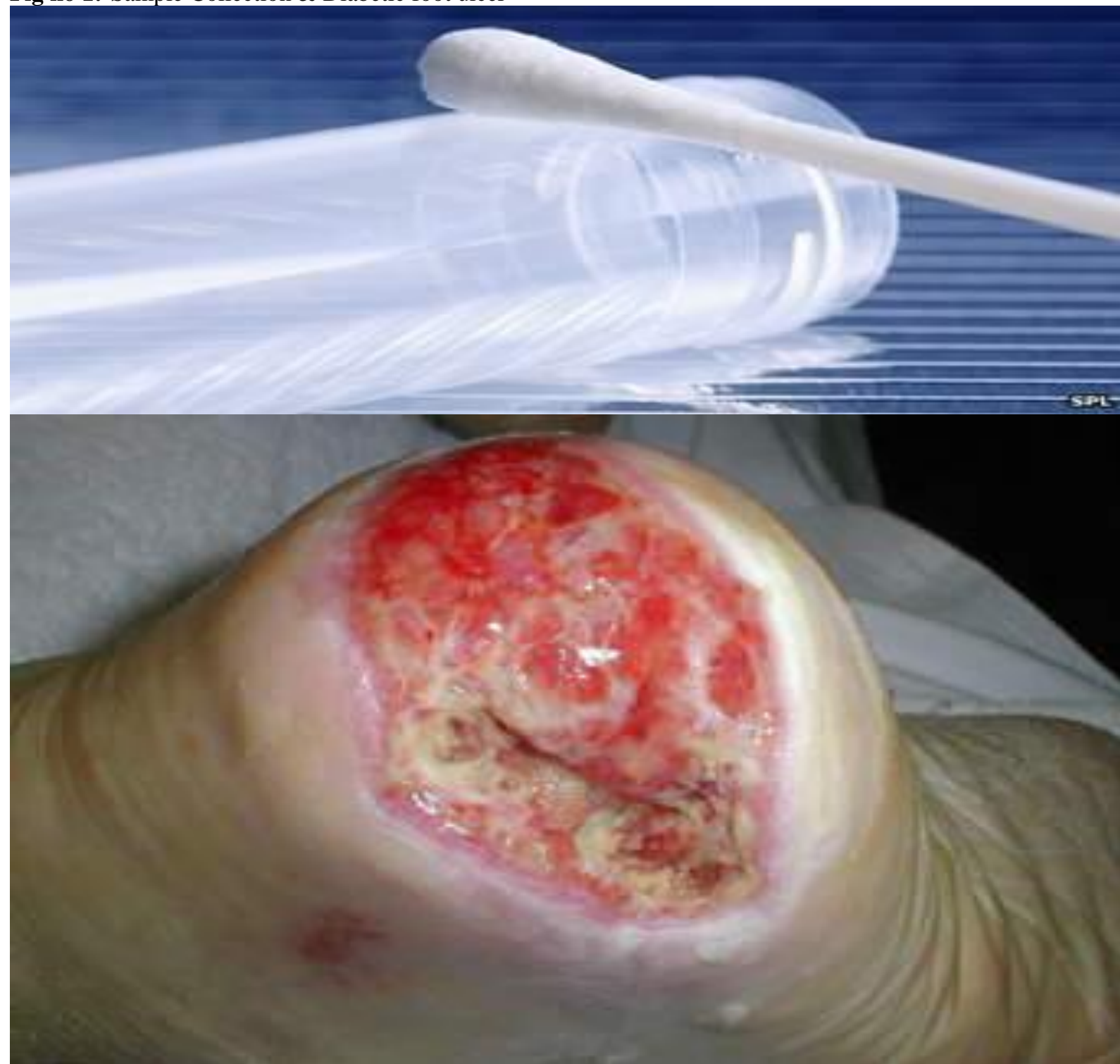

Table no 1:-Age and sex distribution of the study population.

\begin{tabular}{l|l|l}
\hline $31-40$ & 9 & 4 \\
\hline
\end{tabular}




\begin{tabular}{|l|l|l|l|}
\hline $41-50$ & 21 & 8 & 29 \\
\hline $51-60$ & 22 & 11 & 33 \\
\hline$>60$ & 20 & 5 & 25 \\
\hline Total & 72 & 28 & 100 \\
\hline
\end{tabular}

Fig no 2:-Organisms isolated in pus culture

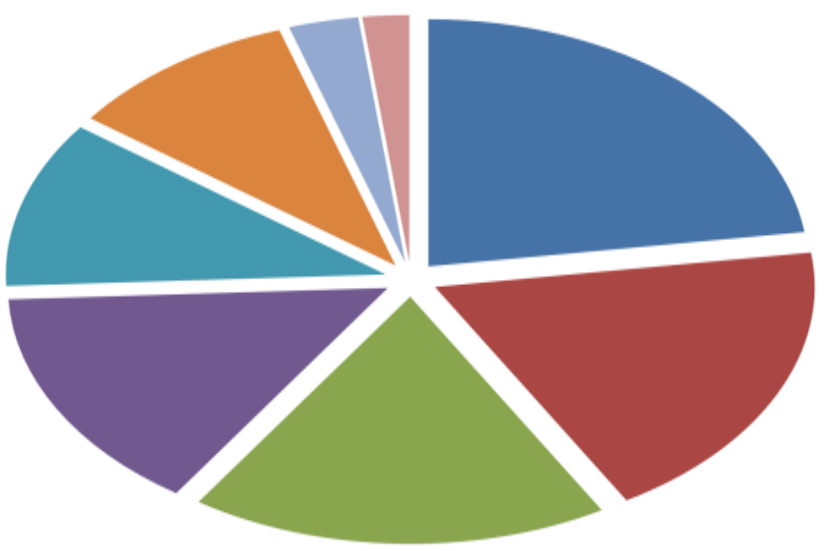

- Pseudomona

E.coli

S.aureus

- Klebsiella

Proteus

CONS

Entericocci

Citrobacter

Fig no 3:-Gram negative bacteria isolates

\section{GNB}

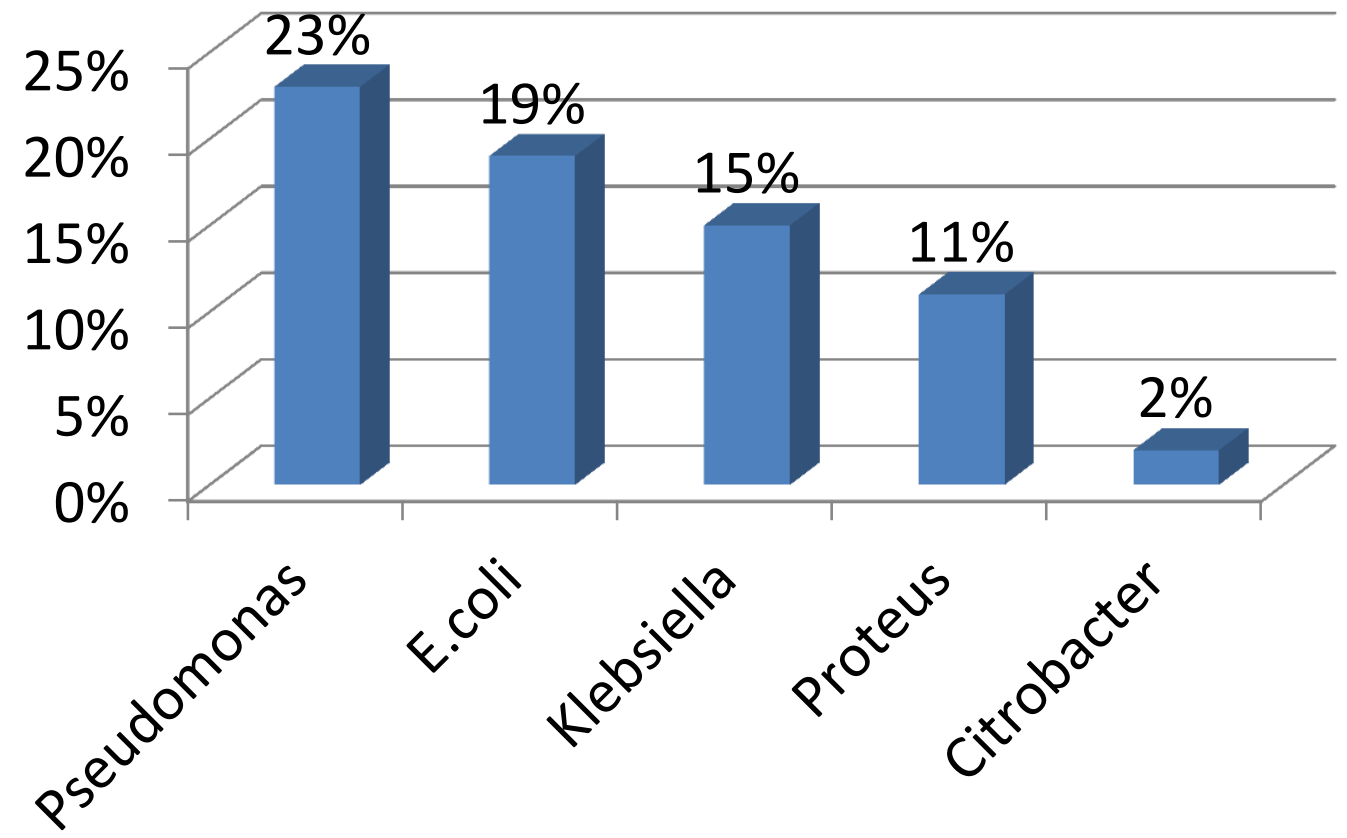


Fig no 4:-Gram positive bacteria isolates

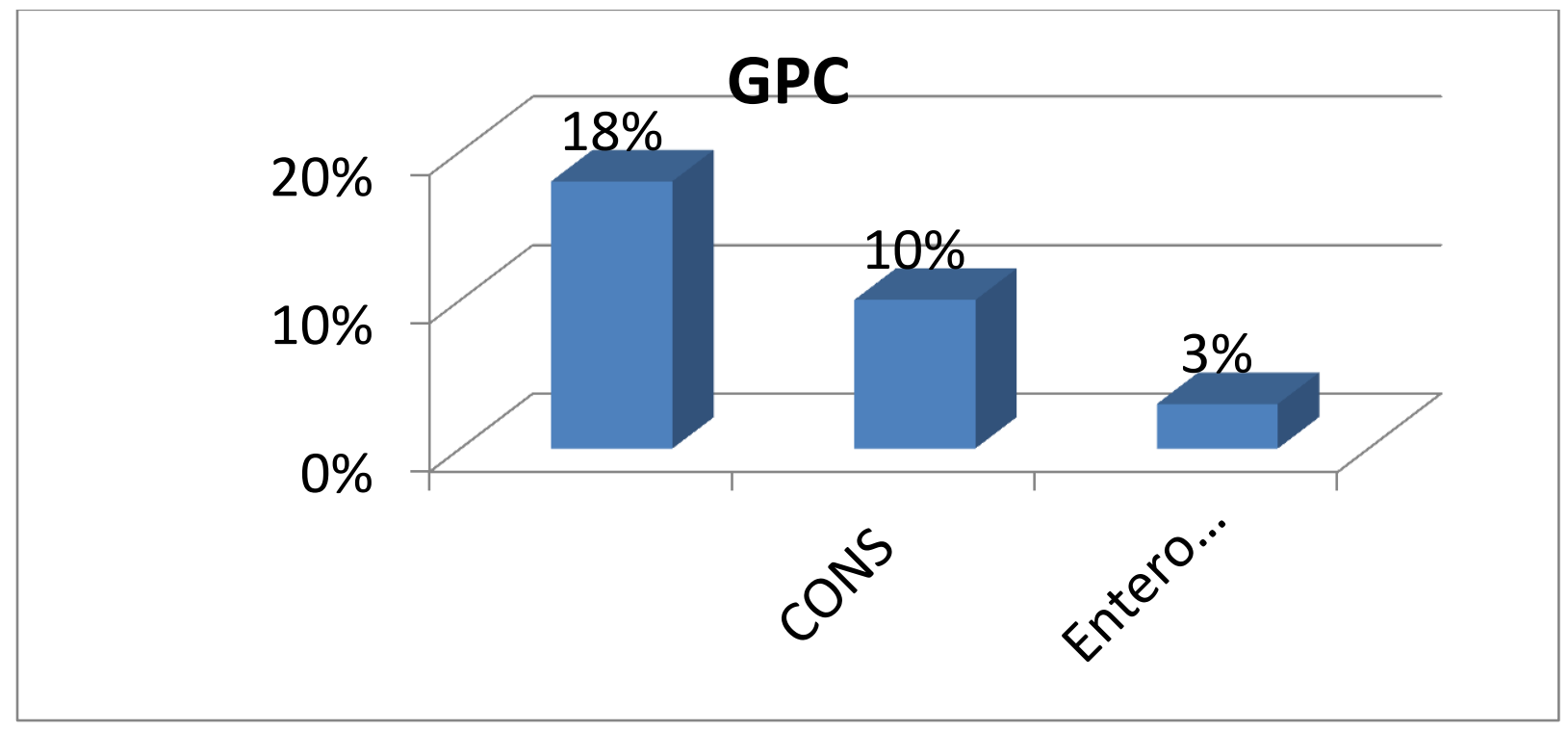

Fig no 5:-Antibiotic susceptibility in Gram negative bacilli

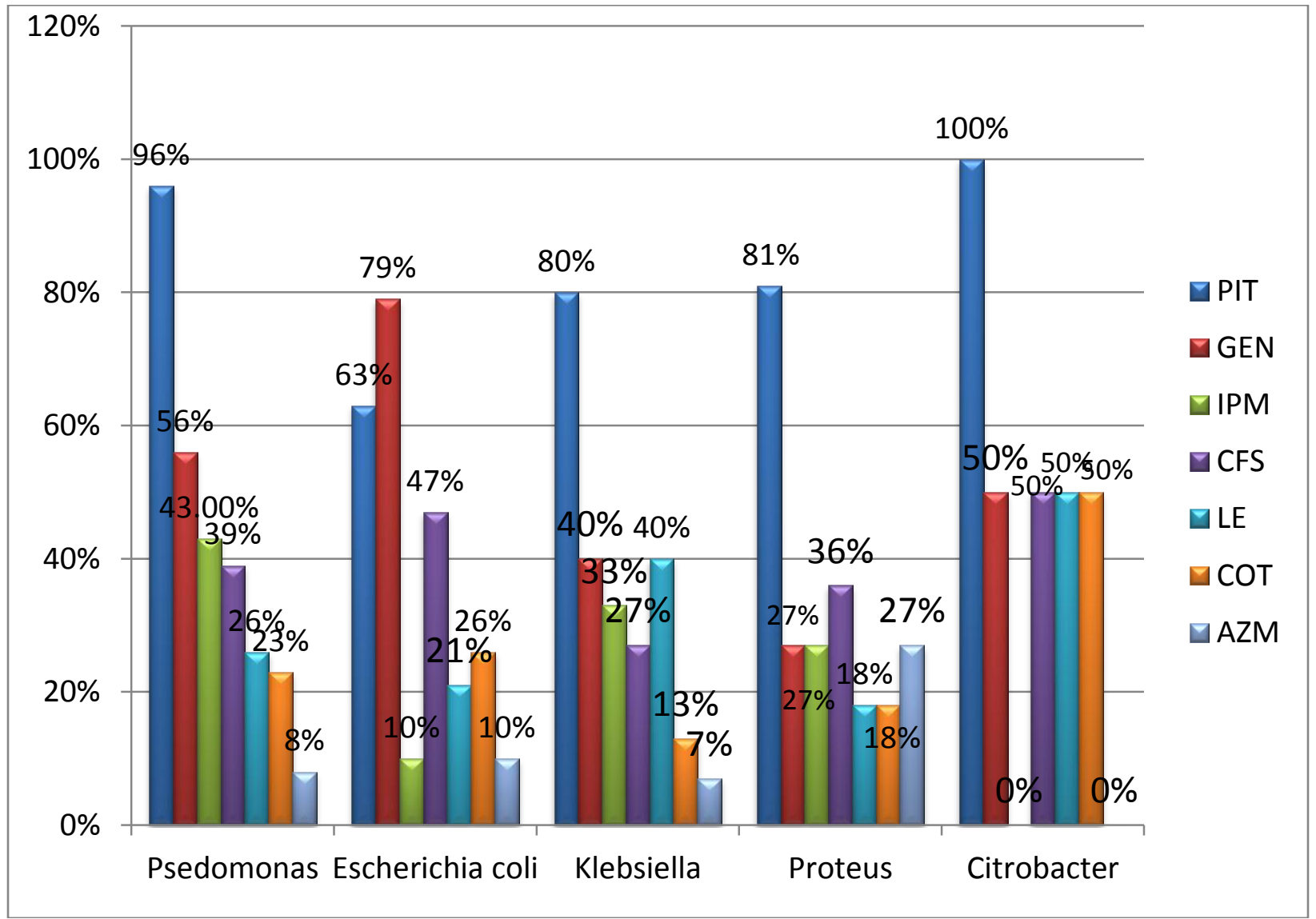

PIT - Piperacillin-tazobactam, GEN - Gentamicin, IPM - Imepenem, CFS - Cefaperzone salbactam, LE - Levofloxacin, COT - Cotrimoxazole, AZM - Azithromycin. 
Fig no 6:-Antibiotic susceptibility in Gram positive cocci

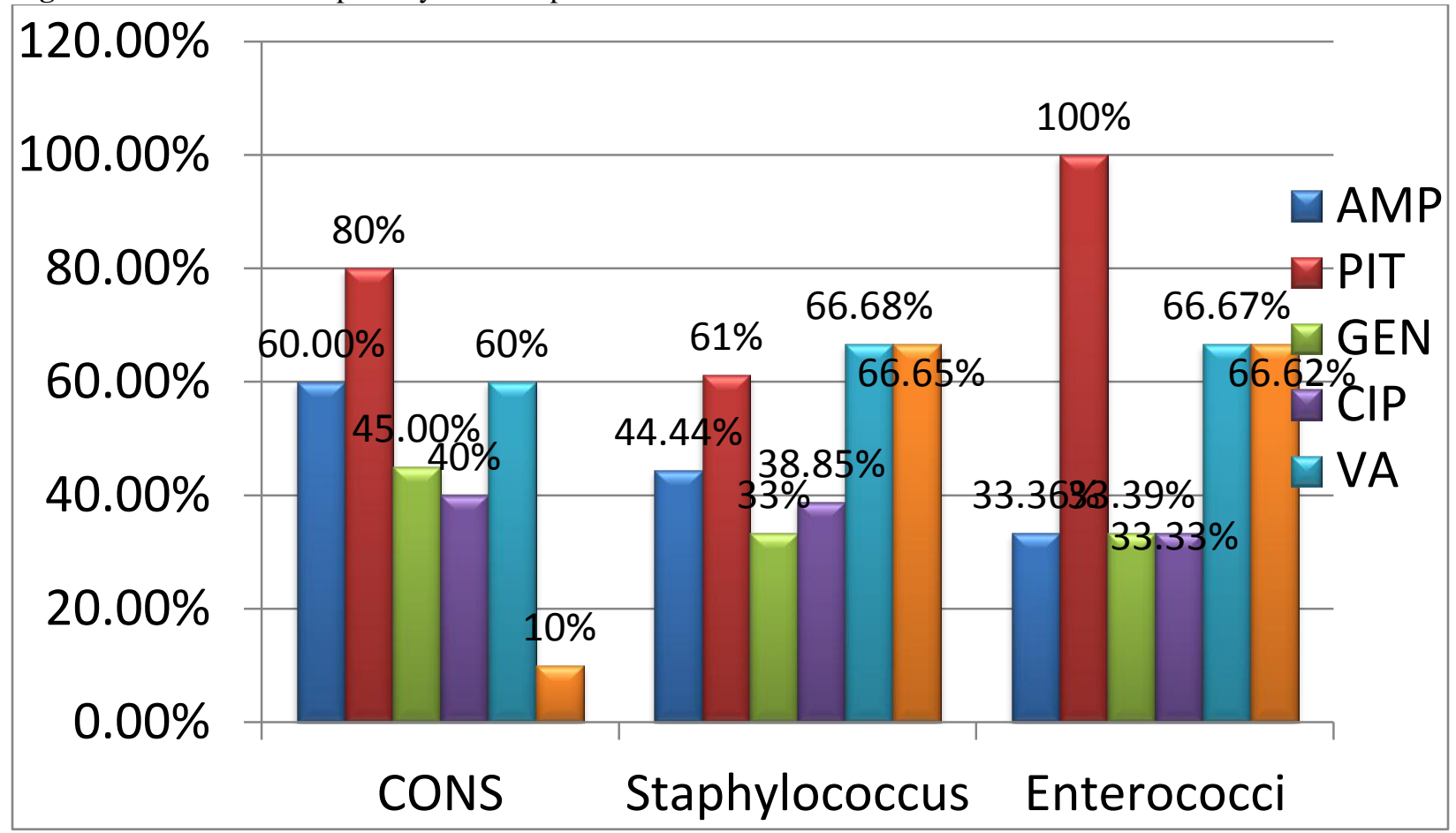

AMP - Ampicillin, PIT - Piperacillin tazobactam, GEN - Gentamicin, CIP - Ciprofloxacin, VA - Vancomycin, LZ - Linezolid.

\section{References:-}

1. Alwin C,Powers Diabetes Mellitus Part 16 Chapter 419.Harrison's Principles of Internal Medicine-vol II $20^{\text {th }}$ edition.

2. Anand A, Biswal I, Soni RK Sinha A, Rynga D, Deb M. A clinic microbiological study of diabetic foot ulcer patients to identify risk factors and their correlation with prognosis in tertiary care hospital in

3. India. Int Surg J 2016

4. Alavi A, Sibbald RG, Mayer D, Goodman L, Botros M, Armstrong DG, et al, Diabetic foot ulcers; Part II. Management J Am Acad Dermatol. 2014.

5. Banashankari G, Rudresh H, Harsha A. Prevalence of gram negative bacteria in diabetic foot-a clinic-microbial study. Al Ameen J Med Sci 2012.

6. Benjamen A L, Anthony RB Paul B. Coronia, et al. Infectious diseases society of America clinical practice guidelines for the diagnosis and treatment of diabetic foot infections. Clinical infectious Dise 2012.

7. Bronze MS,Khardhori R,editor .diabetic foot infections treatment and management Medscape;2016. Available from: http://emedicine.medscape.com

8. Chaudhry WN, Badar R, Jamal M, Jeong J, Zafar J, Andleeb S. Clinico-microbiological study and antibiotic resistance profile of meca and ESBL Gene Prevalence in patients with diabetic foot infections. Exp Ther Med.2016 March.11.

9. Chahine EB. Diabetic foot infections; An update on treatment. US Pharm 2013.

10. Dr Amit Kumar C jain a New classification of diabetic foot complications: a simple and effective teaching tool. The journal of Diabetic Foot Complications, 2012; Volume 4.

11. Edmonds M. Diabetic Foot Ulcer: Practical treatment recommendations. Drugs 2006;66.

12. George A, Dan AA,Eapen BA, ChaithraS, Sivakumar TA. Prospective study on Bacteriology and Prescribing Pattern of Antibiotics in Diabetic Foot Ulcers at Teritiary care Teaching Hospital.WJPMR 2011.

13. Gadepalli R, Dhawan B, Sreenivas V, et al. A clinic microbiological study of diabetic foot ulcers in an Indian tertiary care hospital. Diabetes Care 2006.

14. Gaur DS, Varma A, Pratima Gupta. Diabetic Foot in Uttaranchal. Jk Sci 2007.

15. Huri HZ. Management of the Diabetic Foot. Continuing Professional Development(CPD:Pharmacy Today (Malaysia), Pharmacy Practice 2014. 
16. Hefni AA, Ibrahim AM, Attia KM, Moawad MM<El-ramah AF, Shahin MM, et al. Bacteriological study of diabetic foot infection in Egypt. J Arab Soc Med Res 2013.

17. Mohammad Zubair, Abida Malik, Jamal Ahmad. Clinico-bacteriology and risk factors for the diabetic foot infection with multidrug resistant microorganisms in north India. Biology Med 2010.

18. Mehta VJ, Kikani KM, Mehta SJ. Microbiological profile of Diabetic foot ulcers and its antibiotic susceptibility pattern in Teaching Hospital, Gujarat.

19. Nadeem Sajjad Raja Microbiology of diabetic foot infections in a teaching hospital in Malaysia: a retrospective study of 194 cases J Microbiol Immunol Infect 2007.

20. Osariemen IJ, Olowu SS, Adevbo E, Omom EE, Victoria O, Imuetinyan EJ, et al. aerobic bacteria associated with diabetic wounds in patients attending clinic in a rural community in Nigeria. Glob Res J Microbial 2013.

21. Kishore S, Upadhyay AD, VP J. awareness of foot care among patients with diabetes attending a tertiary care hospital. Natl Med J India 2015.

22. Kali A, Srirangaraj S, Kumar S, Divya HA Kalyani A, Umadevi S. Detection of metallo-beta-lactamase producing Pseudomonas aeruginosa in intensive care units. The Australasian Medical Journal. 2013.

23. Pappu AK, Sinha A, Johnson A. Microbiological profile of diabetic foot ulcer. Calicut Med J 2011.

24. Sivaraman U, Shailesh K, Noyal MJ et al. Microbiological study of diabetic foot infections. Indian J Medical Specialities 2011.

25. World HealthDay 2016:Diabetes, Available

http;//www.searo.who.int/india/mediacentre/events/2016en/Acessed on 20 December 2016.

26. Yazdanpanah L, NasiriM< Adarvishi M. literture review on the management of diabetic foot ulcer. World $\mathbf{J}$ Diabetes .2015. 